OECD Economics Department Working Papers No. 1182

The Heterogeneity of Product Market

\author{
Jean-Marc Fournier
}

Regulations 
Organisation de Coopération et de Développement Économiques

Organisation for Economic Co-operation and Development

05-Jan-2015

ECONOMICS DEPARTMENT

English - Or. English

\section{THE HETEROGENEITY OF PRODUCT MARKET REGULATIONS}

ECONOMICS DEPARTMENT WORKING PAPERS No. 1182

By Jean-Marc Fournier

OECD Working Papers should not be reported as representing the official views of the OECD or of its member countries. The opinions expressed and arguments employed are those of the author(s).

Authorised for publication by Robert Ford, Deputy-Director, Country Studies Branch, Economics Department.

All Economics Department Working Papers are available through OECD's Internet website at www.oecd.org/eco/workingpapers

Complete document available on OLIS in its original format

This document and any map included herein are without prejudice to the status of or sovereignty over any territory, to the delimitation of international frontiers and boundaries and to the name of any territory, city or area. 
OECD Working Papers should not be reported as representing the official views of the OECD or of its member countries. The opinions expressed and arguments employed are those of the author(s).

Working Papers describe preliminary results or research in progress by the author(s) and are published to stimulate discussion on a broad range of issues on which the OECD works.

Comments on Working Papers are welcomed, and may be sent to OECD Economics Department, 2 rue André-Pascal, 75775 Paris Cedex 16, France, or by e-mail to eco.contact@ oecd.org

This document and any map included herein are without prejudice to the status of or sovereignty over any territory, to the delimitation of international frontiers and boundaries and to the name of any territory, city or area.

The statistical data for Israel are supplied by and under the responsibility of the relevant Israeli authorities. The use of such data by the OECD is without prejudice to the status of the Golan Heights, East Jerusalem and Israeli settlements in the West Bank under the terms of international law. 


\section{ABSTRACT/RÉSUMÉ \\ The Heterogeneity of Product Market Regulations}

This paper is making use of the OECD product market regulation (PMR) database to measure the heterogeneity of product market regulation across countries for the whole economy, for the main subcomponents of the PMR indicator and for the internet economy. The heterogeneity within EU countries is quite close to the heterogeneity between EU and non-EU countries. Reforms are associated in a majority of cases with a reduction of heterogeneity, and heterogeneity in countries has diminished more the farther they were from common practices.

This Working Paper relates to the 2014 OECD Economic Survey of the European Union (www.oecd.org/eco/surveys/economic-survey-european-union.htm).

\section{JEL classification: F15, K20.}

Key words: product market regulation, heterogeneity, trade

*********************************************

\section{L'hétérogénéité de la réglementation des marchés de produits}

Ce document utilise la base de données de la réglementation des marchés de produits de l'OCDE (RMP) pour mesurer l'hétérogénéité entre pays de la réglementation des marchés de produits pour l'ensemble de l'économie, pour les principales sous-composantes de l'indicateur RMP et pour l'économie de l'internet. L'hétérogénéité entre pays de l'UE est assez proche de l'hétérogénéité entre les pays de l'UE et les pays hors de l'UE. Les réformes sont associées dans la majorité des cas à une réduction de l'hétérogénéité, et l'hétérogénéité dans les pays a diminué davantage lorsque qu'ils étaient plus loin des pratiques courantes.

Ce Document de travail a trait à l'Étude économique de l'OCDE de l'Union européenne, 2014 (www.oecd.org/fr/eco/etudes/etude-economique-union-europeenne).

Classification JEL : F15, K20.

Mots clefs : réglementation des marchés de produits, hétérogénéité, commerce 
TABLE OF CONTENTS

THE HETEROGENEITY OF PRODUCT MARKET REGULATIONS …...................................................

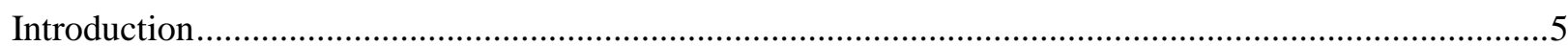

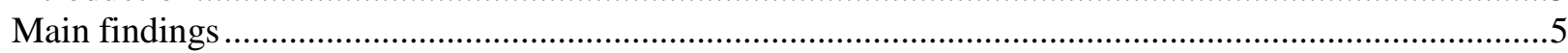

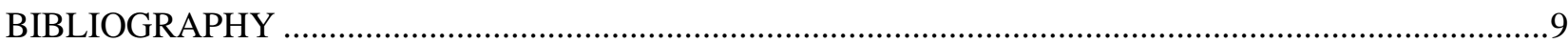

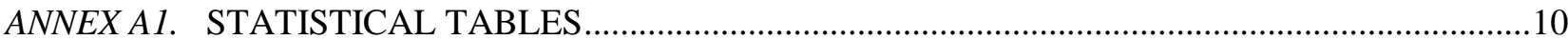

\section{Tables}

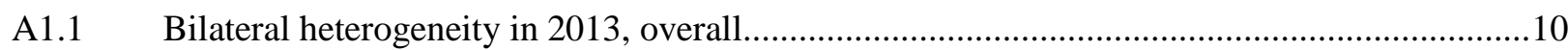

A1.2 Bilateral heterogeneity in 2013, barriers to entrepreneurship ..............................................11

A1.3 Bilateral heterogeneity in 2013, barriers to trade and investment ........................................12

A1.4 Bilateral heterogeneity within EU countries in 2013, state control ......................................13

A1.5 Overall heterogeneity: descriptive statistics by OECD countries...........................................14

A1.6 Bilateral heterogeneity within EU countries in the internet economy .....................................15

\section{Figures}

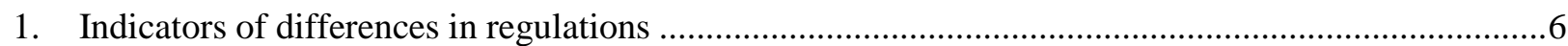

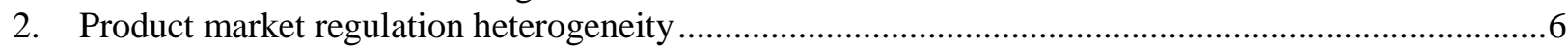

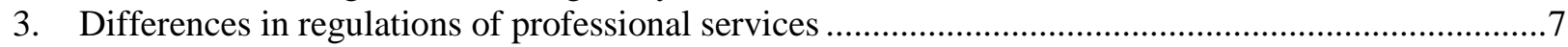

4. Most countries that ease regulations move toward common practices ............................................

5. Heterogeneity in countries diminishes more the farther they are from common practices ..................8

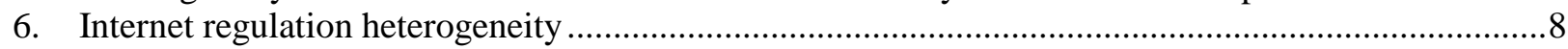


ECO/WKP(2014)78

\title{
THE HETEROGENEITY OF PRODUCT MARKET REGULATIONS
}

\author{
By Jean-Marc Fournier ${ }^{1}$
}

\section{Introduction}

The paper presents evidence on the heterogeneity of product market regulation, with a focus on heterogeneity within the EU. Regulatory heterogeneity is used here to describe heterogeneity within the EU relative to heterogeneity within the whole OECD, to investigate linkages with potential determinants of heterogeneity, to assess the role of past regulatory reforms on heterogeneity. Regulatory heterogeneity can have an adverse impact on firms' cross border activities.

The analysis is based on the OECD Product Market Regulation database, deriving measures of bilateral heterogeneity of regulation across countries. The database uses more than 700 questions to compute the PMR indicator set, which comprises three high-level components: state control, barriers to entrepreneurship, and barriers to foreign trade and investment (Koske et al., 2015).

The bilateral heterogeneity of regulation is measured as the share of regulatory settings that differ between two countries. The methodology is similar to the one used by Kox and Lejour (2006) for services regulations, and by Nordås and Kox (2009) who make use of the World Bank Doing Business indicators. For each question considered in the questionnaire, a country pair is assigned the value 1 if the answer is different and 0 otherwise. These results are averaged with the same weights as in the overall economy PMR indicator. For a given country, its heterogeneity with each other OECD country can be computed, and an average of each of these bilateral measures provides insight how far the given country differs from other OECD countries. The resulting economy-wide measure is a lower bound of regulatory heterogeneity in the sense that even when the answer is the same, regulation can differ for some aspects that are not captured in this questionnaire (e.g. a different design to implement the same regulatory setting).

Two subcomponents of the state control component, namely the scope of state-owned enterprises and direct control over business enterprises (e.g. existence of government's golden shares), do not cover regulations for which heterogeneity directly affects cross-border activities of firms. These subcomponents are thus excluded from the computation of bilateral regulatory heterogeneity.

\section{Main findings}

Heterogeneity among countries has been reduced since a peak in 2003 (Figure 1, left panel). Heterogeneity has increased between 1998 and 2003 because of a rise in barriers to entrepreneurship heterogeneity and, to a lesser extent, because of a rise in heterogeneity of the state control component. The heterogeneity reduction between 2003 and 2008 is mainly driven by a reduction in heterogeneity of barriers to trade and investment, while the reduction between 2008 and 2013 is broad-based.

1. Jean Marc Fournier works in the OECD Economics Department. This paper was originally prepared for the OECD Economic Survey of the European Union published in April 2014 under the authority of the Economic and Development Review Committee. The author is grateful to Robert Ford, Piritta Sorsa, Eckhard Wurzel and Hildegunn Nordås for their helpful comments on earlier drafts. Special thanks go to Isabelle Duong for statistical assistance and Dierdre Claassen and Anthony Bolton for general administrative support. 
The heterogeneity between EU countries that are member of the OECD is smaller than heterogeneity within the OECD overall. There are exceptions however; for instance the heterogeneity in retail trade regulations is higher within the EU than within the OECD since 2008 (Figure 1, right panel). Belgium, the Netherlands and Poland are the closest to other EU countries, while Portugal, Slovenia and Spain are the furthest away from other EU countries (Figure 2). However, these countries do not differ much from the OECD average: the difference of heterogeneity across countries is small. Table A1.1 shows bilateral heterogeneity within the EU for both the economy-wide scope of the PMR indicator and table A1.2 to table A1.4 for its three main subcomponents. Table A1.5 reports a summary of descriptive statistics of heterogeneity within the OECD.

Figure 1. Indicators of differences in regulations

Share of different regulations ${ }^{1}$
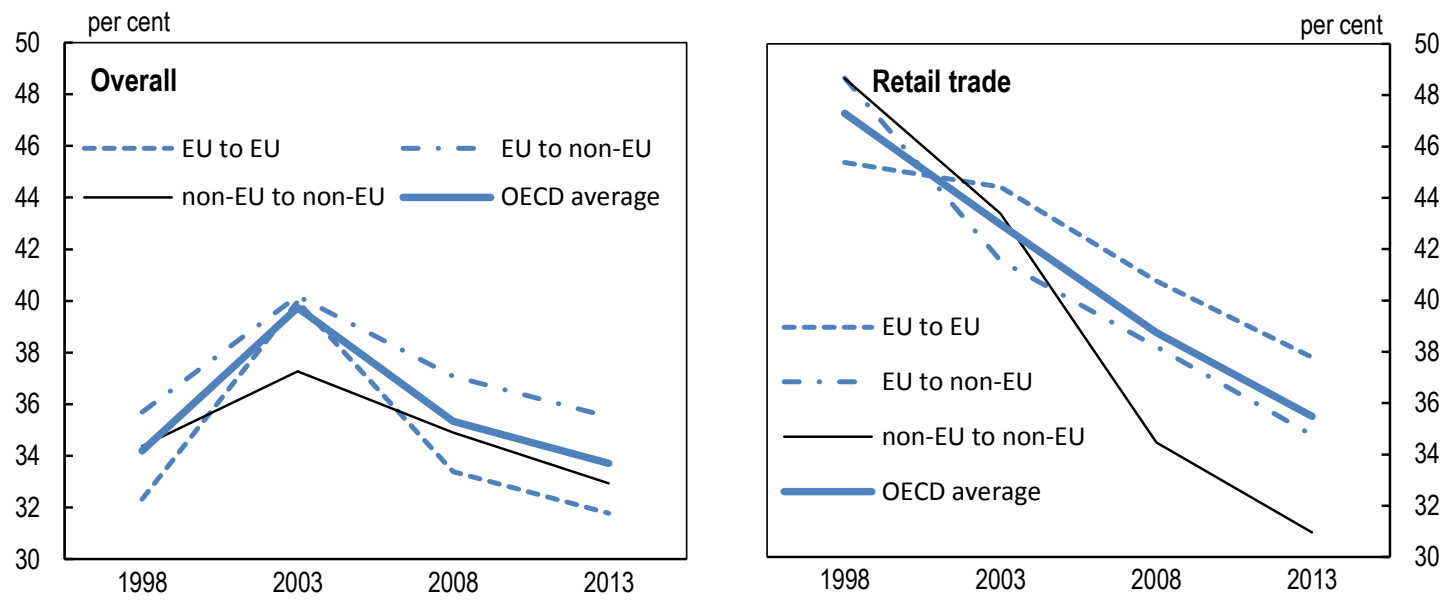

1. EU refers to the $21 \mathrm{EU}$ countries that are member of the OECD.

Source: OECD, Product Market Regulation database and OECD calculations.

Figure 2. Product market regulation heterogeneity

Average bilateral heterogeneity, 2013, per cent

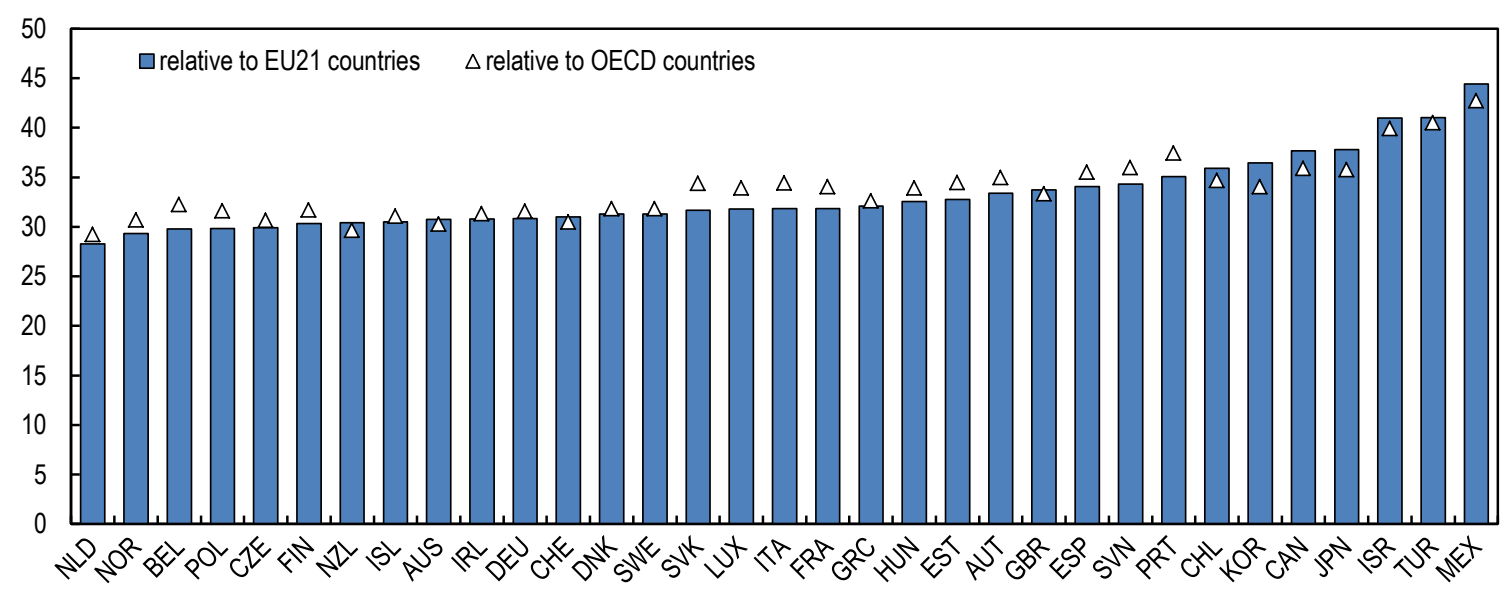

Source: OECD, Product Market Regulation database and OECD calculations.

The correlation between geographical distance and regulatory dissimilarity is positive, but very small. When grouping countries by legal systems (Common Law, French Law, German Law and Nordic Law) the group of Nordic Law countries is the only one that appears to be more homogenous. 
Analysis at the main subcomponent level of the PMR indicator reveals that the heterogeneity of regulations directly related to trade and investment is small, while rules about state control and barriers to entrepreneurship are more heterogeneous (Table A1.2 to table A1.4). Looking more into details allows investigating further the heterogeneity patterns in fields that are important for the EU Single Market, such as regulations on professional services. Restrictions within the EU in this field are only slightly more similar than between EU countries and other OECD countries and heterogeneity has little decreased since 1998 (Figure 3).

Figure 3. Differences in regulations of professional services

Share of different regulations

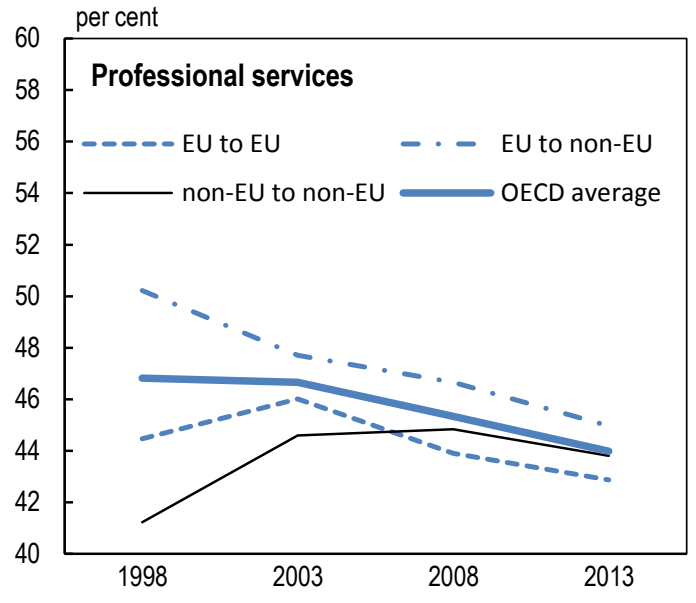

Source: OECD, Product Market Regulation database and OECD calculations

The effect of each country's reforms on heterogeneity can be assessed by comparing the actual average heterogeneity of a given country to the average heterogeneity of this country if it had kept regulations unchanged between 2008 and 2013, while other countries are reforming. 14 out of 19 countries that eased regulatory settings on average since 2008 have also moved toward common practices (Figure 4). Countries that were the furthest away from common practices reduced heterogeneity most (Figure 5).

Figure 4. Most countries that ease regulations move toward common practices ${ }^{1}$

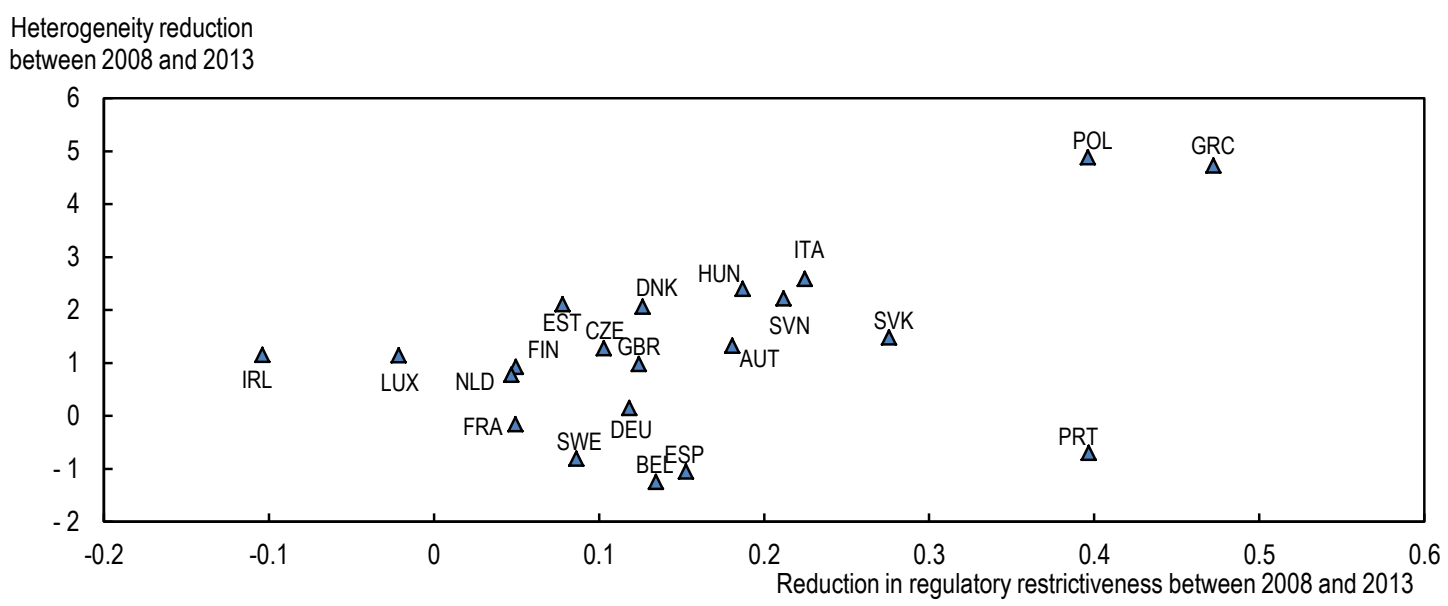

1. The vertical axis shows the difference between the average bilateral heterogeneity of a given country in 2013 and the average bilateral heterogeneity that would be observed if no change had been made in this country's regulations between 2008 and 2013. Source: OECD, Product Market Regulation database and OECD calculations. 
Figure 5. Heterogeneity in countries diminishes more the farther they are from common practices

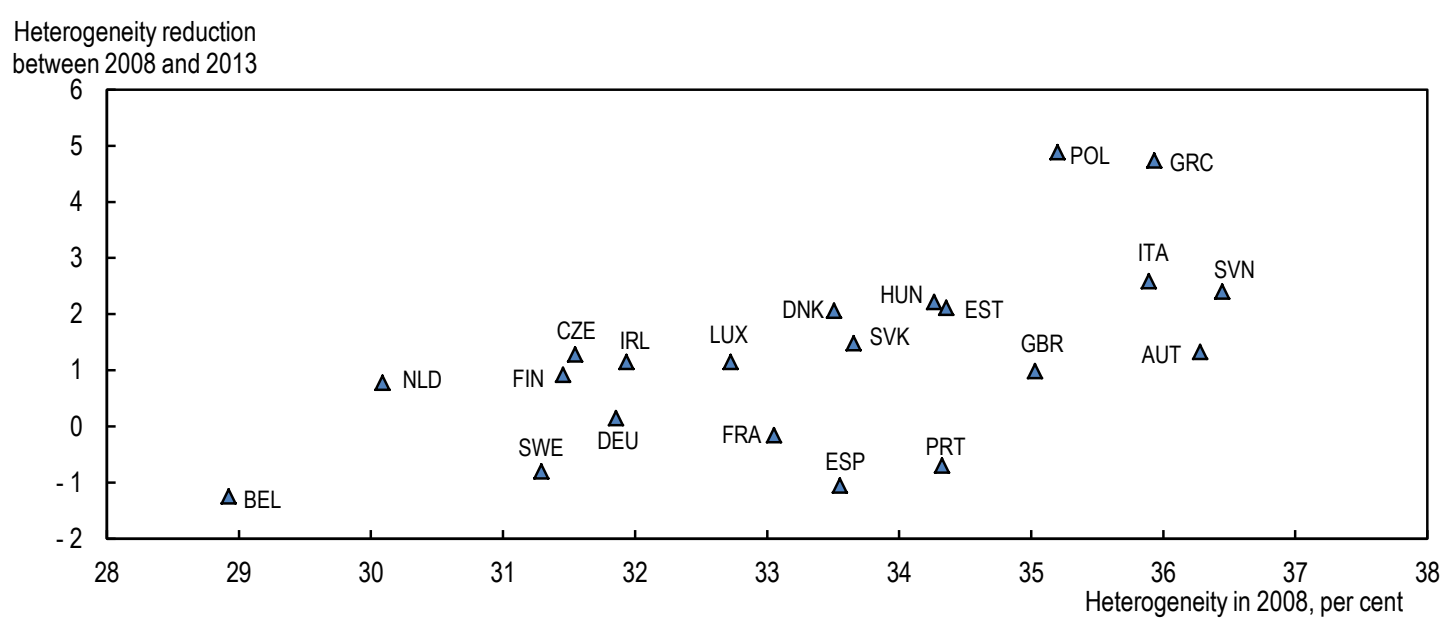

1. The vertical axis shows the difference between the average bilateral heterogeneity of a given country in 2013 and the average bilateral heterogeneity that would be observed if no change had been made in this country's regulations between 2008 and 2013. Source: OECD, Product Market Regulation database and OECD calculations.

Additional questions of the PMR questionnaire (that are not used to compute the PMR indicator set) can be used to analyse countries' regulatory stance towards the Internet economy (Koske et al., 2014). An extension of the analysis to the internet economy is made on top on the economy-wide indicator, making use of equal weights for all questions. Results show that the heterogeneity within the EU is roughly similar to the heterogeneity within the OECD (see Figure 6 and Table A1.6).

Figure 6. Internet regulation heterogeneity

Average bilateral heterogeneity, 2013, per cent

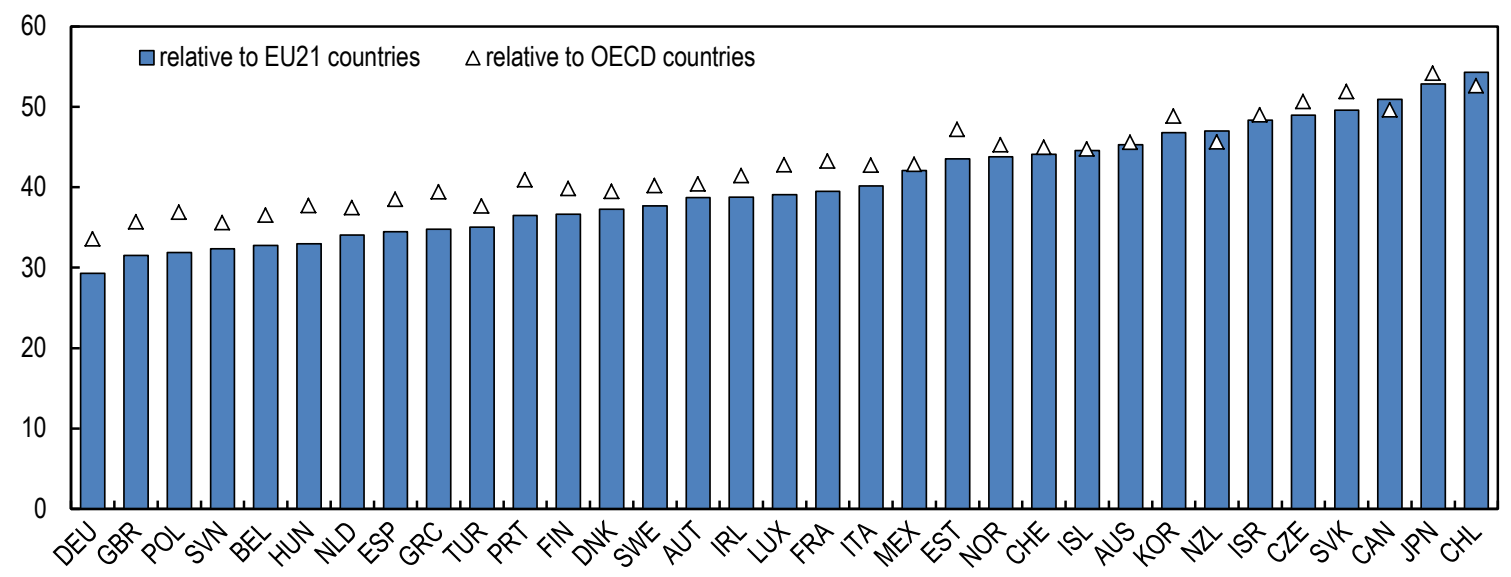

Source: OECD, Product Market Regulation database and OECD calculations. 


\section{Bibliography}

Koske, I., R. Bitetti, I.Wanner and E. Sutherland (2014), “The Internet Economy: Regulatory Challenges and Practices", OECD Economics Department Working Papers, No. 1171, OECD Publishing, Paris.

Koske, I., I.Wanner, R. Bitetti and O. Barbiero (2015), "The 2013 update of the OECD product market regulation indicators: policy insights for OECD and non-OECD countries", OECD Economics Department Working Papers, forthcoming.

Kox, H. and A. Lejour (2006), "The Effects of the Services Directive on Intra-EU Trade and FDI", Presses de Sciences Po, Revue économique, No. 4, Vol. 57, pp. 747-769.

Nordås, H. K. and H. Kox (2009), "Quantifying Regulatory Barriers to Services Trade”, OECD Trade Policy Papers, No. 85, OECD Publishing, Paris. 
ANNEX Al.

STATISTICAL TABLES

Table A1.1. Bilateral heterogeneity within EU countries in 2013, overall

\begin{tabular}{|c|c|c|c|c|c|c|c|c|c|c|c|c|c|c|c|c|c|c|c|c|c|}
\hline & AUT & BEL & CZE & DNK & EST & FIN & FRA & DEU & GRC & HUN & IRL & ITA & LUX & NLD & POL & PRT & SVK & SVN & ESP & SWE & GBR \\
\hline AUT & 0.00 & 0.31 & 0.29 & 0.35 & 0.38 & 0.36 & 0.31 & 0.27 & 0.31 & 0.34 & 0.35 & 0.35 & 0.35 & 0.31 & 0.29 & 0.34 & 0.34 & 0.37 & 0.31 & 0.38 & 0.36 \\
\hline BEL & 0.31 & 0.00 & 0.30 & 0.31 & 0.31 & 0.28 & 0.27 & 0.30 & 0.29 & 0.23 & 0.29 & 0.29 & 0.31 & 0.31 & 0.27 & 0.29 & 0.29 & 0.34 & 0.33 & 0.30 & 0.34 \\
\hline CZE & 0.29 & 0.30 & 0.00 & 0.32 & 0.33 & 0.31 & 0.30 & 0.26 & 0.31 & 0.35 & 0.28 & 0.32 & 0.32 & 0.22 & 0.27 & 0.37 & 0.28 & 0.29 & 0.32 & 0.23 & 0.29 \\
\hline DNK & 0.35 & 0.31 & 0.32 & 0.00 & 0.35 & 0.28 & 0.31 & 0.26 & 0.29 & 0.28 & 0.31 & 0.29 & 0.33 & 0.25 & 0.35 & 0.40 & 0.33 & 0.36 & 0.32 & 0.29 & 0.30 \\
\hline FIN & 0.36 & 0.28 & 0.31 & 0.28 & 0.30 & 0.00 & 0.32 & 0.27 & 0.31 & 0.33 & 0.25 & 0.31 & 0.28 & 0.25 & 0.30 & 0.33 & 0.31 & 0.36 & 0.33 & 0.29 & 0.30 \\
\hline FRA & 0.31 & 0.27 & 0.30 & 0.31 & 0.37 & 0.32 & 0.00 & 0.38 & 0.33 & 0.30 & 0.33 & 0.28 & 0.33 & 0.28 & 0.28 & 0.30 & 0.31 & 0.32 & 0.34 & 0.34 & 0.38 \\
\hline DEU & 0.27 & 0.30 & 0.26 & 0.26 & 0.33 & 0.27 & 0.38 & 0.00 & 0.28 & 0.32 & 0.28 & 0.36 & 0.31 & 0.25 & 0.33 & 0.35 & 0.32 & 0.39 & 0.30 & 0.28 & 0.31 \\
\hline GRC & 0.31 & 0.29 & 0.31 & 0.29 & 0.36 & 0.31 & 0.33 & 0.28 & 0.00 & 0.33 & 0.34 & 0.34 & 0.30 & 0.28 & 0.29 & 0.38 & 0.35 & 0.35 & 0.32 & 0.32 & 0.32 \\
\hline IRL & 0.35 & 0.29 & 0.28 & 0.31 & 0.30 & 0.25 & 0.33 & 0.28 & 0.34 & 0.37 & 0.00 & 0.34 & 0.31 & 0.23 & 0.28 & 0.36 & 0.33 & 0.35 & 0.31 & 0.27 & 0.26 \\
\hline ITA & 0.35 & 0.29 & 0.32 & 0.29 & 0.29 & 0.31 & 0.28 & 0.36 & 0.34 & 0.31 & 0.34 & 0.00 & 0.33 & 0.30 & 0.28 & 0.34 & 0.25 & 0.37 & 0.32 & 0.35 & 0.35 \\
\hline LUX & 0.35 & 0.31 & 0.32 & 0.33 & 0.27 & 0.28 & 0.33 & 0.31 & 0.30 & 0.33 & 0.31 & 0.33 & 0.00 & 0.27 & 0.30 & 0.36 & 0.29 & 0.30 & 0.39 & 0.34 & 0.34 \\
\hline NLD & 0.31 & 0.31 & 0.22 & 0.25 & 0.32 & 0.25 & 0.28 & 0.25 & 0.28 & 0.33 & 0.23 & 0.30 & 0.27 & 0.00 & 0.27 & 0.32 & 0.29 & 0.31 & 0.30 & 0.29 & 0.28 \\
\hline POL & 0.29 & 0.27 & 0.27 & 0.35 & 0.31 & 0.30 & 0.28 & 0.33 & 0.29 & 0.30 & 0.28 & 0.28 & 0.30 & 0.27 & 0.00 & 0.29 & 0.27 & 0.31 & 0.35 & 0.31 & 0.29 \\
\hline PRT & 0.34 & 0.29 & 0.37 & 0.40 & 0.38 & 0.33 & 0.30 & 0.35 & 0.38 & 0.32 & 0.36 & 0.34 & 0.36 & 0.32 & 0.29 & 0.00 & 0.32 & 0.37 & 0.37 & 0.37 & 0.44 \\
\hline SVK & 0.34 & 0.29 & 0.28 & 0.33 & 0.34 & 0.31 & 0.31 & 0.32 & 0.35 & 0.29 & 0.33 & 0.25 & 0.29 & 0.29 & 0.27 & 0.32 & 0.00 & 0.34 & 0.36 & 0.34 & 0.37 \\
\hline SVN & 0.37 & 0.34 & 0.29 & 0.36 & 0.29 & 0.36 & 0.32 & 0.39 & 0.35 & 0.37 & 0.35 & 0.37 & 0.30 & 0.31 & 0.31 & 0.37 & 0.34 & 0.00 & 0.36 & 0.30 & 0.43 \\
\hline ESP & 0.31 & 0.33 & 0.32 & 0.32 & 0.37 & 0.33 & 0.34 & 0.30 & 0.32 & 0.35 & 0.31 & 0.32 & 0.39 & 0.30 & 0.35 & 0.37 & 0.36 & 0.36 & 0.00 & 0.36 & 0.40 \\
\hline SWE & 0.38 & 0.30 & 0.23 & 0.29 & 0.27 & 0.29 & 0.34 & 0.28 & 0.32 & 0.36 & 0.27 & 0.35 & 0.34 & 0.29 & 0.31 & 0.37 & 0.34 & 0.30 & 0.36 & 0.00 & 0.30 \\
\hline
\end{tabular}

Note: The top $10 \%$ cells are reported in bold. 
Table A1.2. Bilateral heterogeneity within EU countries in 2013, barriers to entrepreneurship

\begin{tabular}{|c|c|c|c|c|c|c|c|c|c|c|c|c|c|c|c|c|c|c|c|c|c|}
\hline & AUT & BEL & CZE & DNK & EST & FIN & FRA & DEU & GRC & HUN & IRL & ITA & LUX & NLD & $\mathrm{POL}$ & PRT & SVK & SVN & $\mathrm{ESP}$ & SWE & GBR \\
\hline AUT & 0.00 & 0.37 & 0.34 & 0.45 & 0.39 & 0.42 & 0.33 & 0.38 & 0.38 & 0.41 & 0.45 & 0.28 & 0.36 & 0.39 & 0.28 & 0.40 & 0.33 & 0.36 & 0.43 & 0.43 & 0.43 \\
\hline BEL & 0.37 & 0.00 & 0.36 & 0.37 & 0.37 & 0.38 & 0.31 & 0.37 & 0.37 & 0.34 & 0.41 & 0.30 & 0.42 & 0.35 & 0.31 & 0.35 & 0.35 & 0.36 & 0.41 & 0.34 & 0.48 \\
\hline CZE & 0.34 & 0.36 & 0.00 & 0.33 & 0.35 & 0.34 & 0.32 & 0.27 & 0.28 & 0.44 & 0.39 & 0.32 & 0.31 & 0.25 & 0.36 & 0.42 & 0.33 & 0.31 & 0.34 & 0.29 & 0.36 \\
\hline DNK & 0.45 & 0.37 & 0.33 & 0.00 & 0.35 & 0.28 & 0.42 & 0.29 & 0.33 & 0.37 & 0.36 & 0.37 & 0.35 & 0.18 & 0.46 & 0.45 & 0.35 & 0.33 & 0.41 & 0.25 & 0.32 \\
\hline FIN & 0.42 & 0.38 & 0.34 & 0.28 & 0.32 & 0.00 & 0.43 & 0.29 & 0.37 & 0.41 & 0.35 & 0.38 & 0.33 & 0.26 & 0.44 & 0.45 & 0.39 & 0.36 & 0.35 & 0.28 & 0.39 \\
\hline FRA & 0.33 & 0.31 & 0.32 & 0.42 & 0.46 & 0.43 & 0.00 & 0.41 & 0.34 & 0.39 & 0.45 & 0.32 & 0.44 & 0.38 & 0.34 & 0.35 & 0.33 & 0.36 & 0.43 & 0.39 & 0.46 \\
\hline DEU & 0.38 & 0.37 & 0.27 & 0.29 & 0.31 & 0.29 & 0.41 & 0.00 & 0.37 & 0.38 & 0.34 & 0.36 & 0.34 & 0.26 & 0.42 & 0.44 & 0.34 & 0.36 & 0.35 & 0.30 & 0.37 \\
\hline GRC & 0.38 & 0.37 & 0.28 & 0.33 & 0.33 & 0.37 & 0.34 & 0.37 & 0.00 & 0.42 & 0.45 & 0.38 & 0.30 & 0.28 & 0.32 & 0.45 & 0.38 & 0.22 & 0.39 & 0.37 & 0.39 \\
\hline IRL & 0.45 & 0.41 & 0.39 & 0.36 & 0.33 & 0.35 & 0.45 & 0.34 & 0.45 & 0.53 & 0.00 & 0.46 & 0.44 & 0.34 & 0.48 & 0.53 & 0.50 & 0.37 & 0.35 & 0.28 & 0.37 \\
\hline ITA & 0.28 & 0.30 & 0.32 & 0.37 & 0.36 & 0.38 & 0.32 & 0.36 & 0.38 & 0.38 & 0.46 & 0.00 & 0.40 & 0.32 & 0.30 & 0.34 & 0.25 & 0.35 & 0.40 & 0.39 & 0.47 \\
\hline LUX & 0.36 & 0.42 & 0.31 & 0.35 & 0.34 & 0.33 & 0.44 & 0.34 & 0.30 & 0.41 & 0.44 & 0.40 & 0.00 & 0.26 & 0.38 & 0.47 & 0.34 & 0.33 & 0.46 & 0.42 & 0.40 \\
\hline NLD & 0.39 & 0.35 & 0.25 & 0.18 & 0.30 & 0.26 & 0.38 & 0.26 & 0.28 & 0.41 & 0.34 & 0.32 & 0.26 & 0.00 & 0.38 & 0.42 & 0.31 & 0.29 & 0.37 & 0.30 & 0.32 \\
\hline POL & 0.28 & 0.31 & 0.36 & 0.46 & 0.36 & 0.44 & 0.34 & 0.42 & 0.32 & 0.39 & 0.48 & 0.30 & 0.38 & 0.38 & 0.00 & 0.40 & 0.34 & 0.27 & 0.44 & 0.38 & 0.43 \\
\hline PRT & 0.40 & 0.35 & 0.42 & 0.45 & 0.45 & 0.45 & 0.35 & 0.44 & 0.45 & 0.37 & 0.53 & 0.34 & 0.47 & 0.42 & 0.40 & 0.00 & 0.34 & 0.41 & 0.45 & 0.42 & 0.57 \\
\hline SVK & 0.33 & 0.35 & 0.33 & 0.35 & 0.39 & 0.39 & 0.33 & 0.34 & 0.38 & 0.30 & 0.50 & 0.25 & 0.34 & 0.31 & 0.34 & 0.34 & 0.00 & 0.41 & 0.47 & 0.43 & 0.48 \\
\hline SVN & 0.36 & 0.36 & 0.31 & 0.33 & 0.26 & 0.36 & 0.36 & 0.36 & 0.22 & 0.43 & 0.37 & 0.35 & 0.33 & 0.29 & 0.27 & 0.41 & 0.41 & 0.00 & 0.39 & 0.28 & 0.37 \\
\hline ESP & 0.43 & 0.41 & 0.34 & 0.41 & 0.40 & 0.35 & 0.43 & 0.35 & 0.39 & 0.52 & 0.35 & 0.40 & 0.46 & 0.37 & 0.44 & 0.45 & 0.47 & 0.39 & 0.00 & 0.34 & 0.47 \\
\hline SWE & 0.43 & 0.34 & 0.29 & 0.25 & 0.28 & 0.28 & 0.39 & 0.30 & 0.37 & 0.45 & 0.28 & 0.39 & 0.42 & 0.30 & 0.38 & 0.42 & 0.43 & 0.28 & 0.34 & 0.00 & 0.32 \\
\hline
\end{tabular}

Note: The top $10 \%$ cells are reported in bold. 
Table A1.3. Bilateral heterogeneity within EU countries in 2013, barriers to trade and investment

\begin{tabular}{|c|c|c|c|c|c|c|c|c|c|c|c|c|c|c|c|c|c|c|c|c|c|}
\hline & AUT & BEL & CZE & DNK & EST & FIN & FRA & DEU & GRC & HUN & $\mathrm{IRL}$ & ITA & LUX & NLD & POL & PRT & SVK & SVN & ESP & SWE & GBR \\
\hline AUT & 0.00 & 0.13 & 0.25 & 0.04 & 0.28 & 0.19 & 0.12 & 0.08 & 0.05 & 0.12 & 0.14 & 0.21 & 0.10 & 0.12 & 0.13 & 0.16 & 0.25 & 0.34 & 0.06 & 0.25 & 0.12 \\
\hline BEL & 0.13 & 0.00 & 0.12 & 0.16 & 0.23 & 0.07 & 0.08 & 0.13 & 0.13 & 0.06 & 0.01 & 0.11 & 0.02 & 0.02 & 0.06 & 0.11 & 0.12 & 0.27 & 0.11 & 0.14 & 0.02 \\
\hline CZE & 0.25 & 0.12 & 0.00 & 0.26 & 0.24 & 0.15 & 0.18 & 0.20 & 0.24 & 0.16 & 0.14 & 0.12 & 0.14 & 0.12 & 0.13 & 0.21 & 0.02 & 0.15 & 0.19 & 0.05 & 0.12 \\
\hline DNK & 0.04 & 0.16 & 0.26 & 0.00 & 0.30 & 0.20 & 0.10 & 0.08 & 0.08 & 0.15 & 0.16 & 0.23 & 0.11 & 0.14 & 0.14 & 0.18 & 0.27 & 0.29 & 0.08 & 0.27 & 0.15 \\
\hline FIN & 0.19 & 0.07 & 0.15 & 0.20 & 0.24 & 0.00 & 0.13 & 0.12 & 0.15 & 0.12 & 0.09 & 0.16 & 0.07 & 0.07 & 0.09 & 0.09 & 0.16 & 0.26 & 0.15 & 0.19 & 0.07 \\
\hline FRA & 0.12 & 0.08 & 0.18 & 0.10 & 0.22 & 0.13 & 0.00 & 0.18 & 0.17 & 0.07 & 0.08 & 0.14 & 0.02 & 0.06 & 0.07 & 0.10 & 0.20 & 0.22 & 0.18 & 0.18 & 0.06 \\
\hline DEU & 0.08 & 0.13 & 0.20 & 0.08 & 0.29 & 0.12 & 0.18 & 0.00 & 0.03 & 0.17 & 0.14 & 0.21 & 0.13 & 0.12 & 0.14 & 0.21 & 0.22 & 0.31 & 0.03 & 0.25 & 0.13 \\
\hline GRC & 0.05 & 0.13 & 0.24 & 0.08 & 0.29 & 0.15 & 0.17 & 0.03 & 0.00 & 0.18 & 0.13 & 0.20 & 0.11 & 0.11 & 0.16 & 0.19 & 0.25 & 0.35 & 0.04 & 0.24 & 0.11 \\
\hline IRL & 0.14 & 0.01 & 0.14 & 0.16 & 0.22 & 0.09 & 0.08 & 0.14 & 0.13 & 0.09 & 0.00 & 0.10 & 0.03 & 0.02 & 0.08 & 0.11 & 0.15 & 0.29 & 0.14 & 0.14 & 0.02 \\
\hline ITA & 0.21 & 0.11 & 0.12 & 0.23 & 0.13 & 0.16 & 0.14 & 0.21 & 0.20 & 0.16 & 0.10 & 0.00 & 0.11 & 0.08 & 0.14 & 0.17 & 0.13 & 0.26 & 0.20 & 0.11 & 0.09 \\
\hline LUX & 0.10 & 0.02 & 0.14 & 0.11 & 0.19 & 0.07 & 0.02 & 0.13 & 0.11 & 0.02 & 0.03 & 0.11 & 0.00 & 0.01 & 0.05 & 0.08 & 0.15 & 0.19 & 0.13 & 0.11 & 0.02 \\
\hline NLD & 0.12 & 0.02 & 0.12 & 0.14 & 0.21 & 0.07 & 0.06 & 0.12 & 0.11 & 0.07 & 0.02 & 0.08 & 0.01 & 0.00 & 0.06 & 0.09 & 0.13 & 0.26 & 0.12 & 0.11 & 0.00 \\
\hline POL & 0.13 & 0.06 & 0.13 & 0.14 & 0.21 & 0.09 & 0.07 & 0.14 & 0.16 & 0.05 & 0.08 & 0.14 & 0.05 & 0.06 & 0.00 & 0.08 & 0.14 & 0.22 & 0.12 & 0.18 & 0.06 \\
\hline PRT & 0.16 & 0.11 & 0.21 & 0.18 & 0.25 & 0.09 & 0.10 & 0.21 & 0.19 & 0.11 & 0.11 & 0.17 & 0.08 & 0.09 & 0.08 & 0.00 & 0.22 & 0.30 & 0.20 & 0.21 & 0.09 \\
\hline SVK & 0.25 & 0.12 & 0.02 & 0.27 & 0.25 & 0.16 & 0.20 & 0.22 & 0.25 & 0.17 & 0.15 & 0.13 & 0.15 & 0.13 & 0.14 & 0.22 & 0.00 & 0.17 & 0.21 & 0.06 & 0.13 \\
\hline SVN & 0.34 & 0.27 & 0.15 & 0.29 & 0.30 & 0.26 & 0.22 & 0.31 & 0.35 & 0.21 & 0.29 & 0.26 & 0.19 & 0.26 & 0.22 & 0.30 & 0.17 & 0.00 & 0.34 & 0.15 & 0.27 \\
\hline ESP & 0.06 & 0.11 & 0.19 & 0.08 & 0.32 & 0.15 & 0.18 & 0.03 & 0.04 & 0.15 & 0.14 & 0.20 & 0.13 & 0.12 & 0.12 & 0.20 & 0.21 & 0.34 & 0.00 & 0.24 & 0.12 \\
\hline SWE & 0.25 & 0.14 & 0.05 & 0.27 & 0.25 & 0.19 & 0.18 & 0.25 & 0.24 & 0.15 & 0.14 & 0.11 & 0.11 & 0.11 & 0.18 & 0.21 & 0.06 & 0.15 & 0.24 & 0.00 & 0.12 \\
\hline
\end{tabular}

Note: The top $10 \%$ cells are reported in bold. 
Table A1.4. Bilateral heterogeneity within EU countries in 2013, state control

\begin{tabular}{|c|c|c|c|c|c|c|c|c|c|c|c|c|c|c|c|c|c|c|c|c|c|}
\hline & AUT & BEL & CZE & DNK & EST & FIN & FRA & DEU & GRC & HUN & IRL & ITA & LUX & NLD & $\mathrm{POL}$ & PRT & SVK & SVN & ESP & SWE & GBR \\
\hline AUT & 0.00 & 0.37 & 0.26 & 0.44 & 0.46 & 0.43 & 0.44 & 0.29 & 0.44 & 0.42 & 0.38 & 0.57 & 0.53 & 0.34 & 0.46 & 0.43 & 0.42 & 0.39 & 0.36 & 0.42 & 0.48 \\
\hline BEL & 0.37 & 0.00 & 0.36 & 0.34 & 0.29 & 0.30 & 0.37 & 0.33 & 0.32 & 0.21 & 0.34 & 0.44 & 0.36 & 0.49 & 0.39 & 0.37 & 0.35 & 0.36 & 0.40 & 0.35 & 0.40 \\
\hline CZE & 0.26 & 0.36 & 0.00 & 0.34 & 0.38 & 0.40 & 0.36 & 0.29 & 0.42 & 0.37 & 0.21 & 0.49 & 0.46 & 0.25 & 0.25 & 0.44 & 0.41 & 0.38 & 0.40 & 0.30 & 0.32 \\
\hline DNK & 0.44 & 0.34 & 0.34 & 0.00 & 0.39 & 0.33 & 0.31 & 0.38 & 0.40 & 0.25 & 0.37 & 0.21 & 0.49 & 0.45 & 0.37 & 0.51 & 0.36 & 0.47 & 0.40 & 0.36 & 0.39 \\
\hline FIN & 0.43 & 0.30 & 0.40 & 0.33 & 0.34 & 0.00 & 0.31 & 0.39 & 0.35 & 0.41 & 0.22 & 0.35 & 0.38 & 0.39 & 0.30 & 0.35 & 0.30 & 0.44 & 0.45 & 0.37 & 0.35 \\
\hline FRA & 0.44 & 0.37 & 0.36 & 0.31 & 0.37 & 0.31 & 0.00 & 0.50 & 0.46 & 0.37 & 0.38 & 0.32 & 0.40 & 0.33 & 0.36 & 0.42 & 0.37 & 0.35 & 0.32 & 0.39 & 0.53 \\
\hline DEU & 0.29 & 0.33 & 0.29 & 0.38 & 0.40 & 0.39 & 0.50 & 0.00 & 0.37 & 0.35 & 0.31 & 0.49 & 0.42 & 0.37 & 0.38 & 0.35 & 0.37 & 0.50 & 0.48 & 0.28 & 0.37 \\
\hline GRC & 0.44 & 0.32 & 0.42 & 0.40 & 0.46 & 0.35 & 0.46 & 0.37 & 0.00 & 0.31 & 0.37 & 0.41 & 0.47 & 0.41 & 0.37 & 0.44 & 0.39 & 0.54 & 0.45 & 0.30 & 0.38 \\
\hline IRL & 0.38 & 0.34 & 0.21 & 0.37 & 0.32 & 0.22 & 0.38 & 0.31 & 0.37 & 0.38 & 0.00 & 0.38 & 0.35 & 0.25 & 0.14 & 0.31 & 0.23 & 0.38 & 0.41 & 0.37 & 0.30 \\
\hline ITA & 0.57 & 0.44 & 0.49 & 0.21 & 0.34 & 0.35 & 0.32 & 0.49 & 0.41 & 0.32 & 0.38 & 0.00 & 0.39 & 0.48 & 0.38 & 0.49 & 0.34 & 0.48 & 0.31 & 0.48 & 0.39 \\
\hline LUX & 0.53 & 0.36 & 0.46 & 0.49 & 0.24 & 0.38 & 0.40 & 0.42 & 0.47 & 0.45 & 0.35 & 0.39 & 0.00 & 0.51 & 0.40 & 0.40 & 0.31 & 0.33 & 0.51 & 0.37 & 0.51 \\
\hline NLD & 0.34 & 0.49 & 0.25 & 0.45 & 0.45 & 0.39 & 0.33 & 0.37 & 0.41 & 0.43 & 0.25 & 0.48 & 0.51 & 0.00 & 0.29 & 0.37 & 0.37 & 0.36 & 0.35 & 0.41 & 0.46 \\
\hline POL & 0.46 & 0.39 & 0.25 & 0.37 & 0.33 & 0.30 & 0.36 & 0.38 & 0.37 & 0.40 & 0.14 & 0.38 & 0.40 & 0.29 & 0.00 & 0.32 & 0.30 & 0.44 & 0.43 & 0.31 & 0.28 \\
\hline PRT & 0.43 & 0.37 & 0.44 & 0.51 & 0.38 & 0.35 & 0.42 & 0.35 & 0.44 & 0.44 & 0.31 & 0.49 & 0.40 & 0.37 & 0.32 & 0.00 & 0.40 & 0.35 & 0.42 & 0.42 & 0.54 \\
\hline SVK & 0.42 & 0.35 & 0.41 & 0.36 & 0.33 & 0.30 & 0.37 & 0.37 & 0.39 & 0.37 & 0.23 & 0.34 & 0.31 & 0.37 & 0.30 & 0.40 & 0.00 & 0.38 & 0.32 & 0.42 & 0.41 \\
\hline SVN & 0.39 & 0.36 & 0.38 & 0.47 & 0.32 & 0.44 & 0.35 & 0.50 & 0.54 & 0.41 & 0.38 & 0.48 & 0.33 & 0.36 & 0.44 & 0.35 & 0.38 & 0.00 & 0.32 & 0.46 & 0.67 \\
\hline ESP & 0.36 & 0.40 & 0.40 & 0.40 & 0.36 & 0.45 & 0.32 & 0.48 & 0.45 & 0.28 & 0.41 & 0.31 & 0.51 & 0.35 & 0.43 & 0.42 & 0.32 & 0.32 & 0.00 & 0.49 & 0.53 \\
\hline SWE & 0.42 & 0.35 & 0.30 & 0.36 & 0.27 & 0.37 & 0.39 & 0.28 & 0.30 & 0.38 & 0.37 & 0.48 & 0.37 & 0.41 & 0.31 & 0.42 & 0.42 & 0.46 & 0.49 & 0.00 & 0.43 \\
\hline
\end{tabular}

Note: The top $10 \%$ cells are reported in bold.

Table 1. 
Table A1.5. Overall heterogeneity: descriptive statistics by OECD countries

\begin{tabular}{|c|c|c|c|c|c|c|c|c|c|c|c|c|c|c|c|c|c|}
\hline & NLD & NZL & AUS & $\mathrm{CHE}$ & CZE & NOR & ISL & IRL & DEU & POL & FIN & SWE & DNK & BEL & GRC & GBR & \\
\hline mean & 0.29 & 0.30 & 0.30 & 0.30 & 0.31 & 0.31 & 0.31 & 0.31 & 0.32 & 0.32 & 0.32 & 0.32 & 0.32 & 0.32 & 0.33 & 0.33 & \\
\hline $\min$ & 0.19 & 0.19 & 0.20 & 0.21 & 0.22 & 0.21 & 0.22 & 0.22 & 0.23 & 0.25 & 0.25 & 0.23 & 0.22 & 0.23 & 0.27 & 0.22 & \\
\hline $\max$ & 0.41 & 0.38 & 0.40 & 0.38 & 0.43 & 0.45 & 0.41 & 0.48 & 0.50 & 0.42 & 0.48 & 0.45 & 0.43 & 0.46 & 0.42 & 0.47 & \\
\hline standard deviation & 0.05 & 0.06 & 0.05 & 0.04 & 0.05 & 0.05 & 0.04 & 0.06 & 0.06 & 0.04 & 0.05 & 0.05 & 0.05 & 0.05 & 0.04 & 0.06 & \\
\hline & LUX & HUN & FRA & KOR & SVK & ITA & EST & $\mathrm{CHL}$ & AUT & ESP & JPN & CAN & SVN & PRT & ISR & TUR & MEX \\
\hline mean & 0.34 & 0.34 & 0.34 & 0.34 & 0.34 & 0.34 & 0.34 & 0.35 & 0.35 & 0.36 & 0.36 & 0.36 & 0.36 & 0.37 & 0.40 & 0.41 & 0.43 \\
\hline $\min$ & 0.27 & 0.23 & 0.27 & 0.22 & 0.25 & 0.25 & 0.27 & 0.28 & 0.27 & 0.30 & 0.22 & 0.22 & 0.29 & 0.29 & 0.34 & 0.33 & 0.31 \\
\hline $\max$ & 0.50 & 0.44 & 0.46 & 0.44 & 0.48 & 0.47 & 0.48 & 0.42 & 0.45 & 0.49 & 0.45 & 0.46 & 0.45 & 0.49 & 0.48 & 0.48 & 0.50 \\
\hline standard deviation & 0.05 & 0.04 & 0.05 & 0.06 & 0.05 & 0.05 & 0.04 & 0.04 & 0.04 & 0.04 & 0.05 & 0.06 & 0.04 & 0.05 & 0.04 & 0.04 & 0.05 \\
\hline
\end{tabular}

Reading Note: For instance, the average bilateral heterogeneity between the Netherland and each other OECD country is 0.29 , with a minimal value of 0.19 . 
Table A1.6. Bilateral heterogeneity within EU countries in the internet economy

\begin{tabular}{|c|c|c|c|c|c|c|c|c|c|c|c|c|c|c|c|c|c|c|c|c|c|}
\hline & AUT & BEL & CZE & DNK & EST & FIN & FRA & DEU & GRC & HUN & IRL & ITA & LUX & NLD & $\mathrm{POL}$ & PRT & SVK & SVN & ESP & SWE & GBR \\
\hline AUT & 0.00 & 0.39 & 0.42 & 0.52 & 0.34 & 0.33 & 0.48 & 0.34 & 0.45 & 0.38 & 0.38 & 0.39 & 0.43 & 0.33 & 0.28 & 0.42 & 0.56 & 0.25 & 0.39 & 0.33 & 0.31 \\
\hline BEL & 0.39 & 0.00 & 0.48 & 0.25 & 0.38 & 0.33 & 0.30 & 0.22 & 0.32 & 0.31 & 0.31 & 0.37 & 0.32 & 0.28 & 0.32 & 0.33 & 0.41 & 0.34 & 0.35 & 0.35 & 0.19 \\
\hline CZE & 0.42 & 0.48 & 0.00 & 0.65 & 0.38 & 0.44 & 0.59 & 0.52 & 0.54 & 0.51 & 0.45 & 0.55 & 0.41 & 0.44 & 0.51 & 0.54 & 0.40 & 0.43 & 0.45 & 0.44 & 0.62 \\
\hline DNK & 0.52 & 0.25 & 0.65 & 0.00 & 0.48 & 0.36 & 0.33 & 0.18 & 0.33 & 0.30 & 0.43 & 0.45 & 0.42 & 0.27 & 0.29 & 0.35 & 0.51 & 0.32 & 0.41 & 0.33 & 0.26 \\
\hline FIN & 0.33 & 0.33 & 0.44 & 0.36 & 0.42 & 0.00 & 0.41 & 0.27 & 0.48 & 0.35 & 0.41 & 0.43 & 0.24 & 0.28 & 0.31 & 0.45 & 0.32 & 0.34 & 0.35 & 0.42 & 0.38 \\
\hline FRA & 0.48 & 0.30 & 0.59 & 0.33 & 0.49 & 0.41 & 0.00 & 0.29 & 0.29 & 0.36 & 0.45 & 0.37 & 0.42 & 0.37 & 0.32 & 0.38 & 0.58 & 0.39 & 0.35 & 0.40 & 0.31 \\
\hline DEU & 0.34 & 0.22 & 0.52 & 0.18 & 0.50 & 0.27 & 0.29 & 0.00 & 0.25 & 0.19 & 0.26 & 0.38 & 0.39 & 0.23 & 0.21 & 0.28 & 0.49 & 0.19 & 0.21 & 0.30 & 0.16 \\
\hline GRC & 0.45 & 0.32 & 0.54 & 0.33 & 0.45 & 0.48 & 0.29 & 0.25 & 0.00 & 0.28 & 0.39 & 0.32 & 0.36 & 0.36 & 0.24 & 0.25 & 0.55 & 0.25 & 0.24 & 0.42 & 0.21 \\
\hline IRL & 0.38 & 0.31 & 0.45 & 0.43 & 0.46 & 0.41 & 0.45 & 0.26 & 0.39 & 0.38 & 0.00 & 0.43 & 0.41 & 0.33 & 0.39 & 0.36 & 0.54 & 0.31 & 0.34 & 0.36 & 0.34 \\
\hline ITA & 0.39 & 0.37 & 0.55 & 0.45 & 0.47 & 0.43 & 0.37 & 0.38 & 0.32 & 0.29 & 0.43 & 0.00 & 0.39 & 0.47 & 0.32 & 0.35 & 0.51 & 0.46 & 0.32 & 0.40 & 0.36 \\
\hline LUX & 0.43 & 0.32 & 0.41 & 0.42 & 0.36 & 0.24 & 0.42 & 0.39 & 0.36 & 0.36 & 0.41 & 0.39 & 0.00 & 0.32 & 0.43 & 0.39 & 0.48 & 0.40 & 0.41 & 0.45 & 0.44 \\
\hline NLD & 0.33 & 0.28 & 0.44 & 0.27 & 0.38 & 0.28 & 0.37 & 0.23 & 0.36 & 0.37 & 0.33 & 0.47 & 0.32 & 0.00 & 0.22 & 0.40 & 0.49 & 0.24 & 0.40 & 0.33 & 0.29 \\
\hline POL & 0.28 & 0.32 & 0.51 & 0.29 & 0.44 & 0.31 & 0.32 & 0.21 & 0.24 & 0.29 & 0.39 & 0.32 & 0.43 & 0.22 & 0.00 & 0.26 & 0.56 & 0.19 & 0.28 & 0.34 & 0.16 \\
\hline PRT & 0.42 & 0.33 & 0.54 & 0.35 & 0.46 & 0.45 & 0.38 & 0.28 & 0.25 & 0.29 & 0.36 & 0.35 & 0.39 & 0.40 & 0.26 & 0.00 & 0.51 & 0.25 & 0.30 & 0.45 & 0.28 \\
\hline SVK & 0.56 & 0.41 & 0.40 & 0.51 & 0.49 & 0.32 & 0.58 & 0.49 & 0.55 & 0.45 & 0.54 & 0.51 & 0.48 & 0.49 & 0.56 & 0.51 & 0.00 & 0.58 & 0.45 & 0.54 & 0.51 \\
\hline SVN & 0.25 & 0.34 & 0.43 & 0.32 & 0.40 & 0.34 & 0.39 & 0.19 & 0.25 & 0.24 & 0.31 & 0.46 & 0.40 & 0.24 & 0.19 & 0.25 & 0.58 & 0.00 & 0.28 & 0.32 & 0.28 \\
\hline ESP & 0.39 & 0.35 & 0.45 & 0.41 & 0.51 & 0.35 & 0.35 & 0.21 & 0.24 & 0.25 & 0.34 & 0.32 & 0.41 & 0.40 & 0.28 & 0.30 & 0.45 & 0.28 & 0.00 & 0.38 & 0.23 \\
\hline SWE & 0.33 & 0.35 & 0.44 & 0.33 & 0.39 & 0.42 & 0.40 & 0.30 & 0.42 & 0.30 & 0.36 & 0.40 & 0.45 & 0.33 & 0.34 & 0.45 & 0.54 & 0.32 & 0.38 & 0.00 & 0.28 \\
\hline
\end{tabular}

Note: The top $10 \%$ cells are reported in bold. 


\section{WORKING PAPERS}

The full series of Economics Department Working Papers can be consulted at www.oecd.org/eco/workingpapers

1181. Implicit regulatory barriers in the EU single market: new empirical evidence from gravity models

(December 2014) by Jean-Marc Fournier, Aurore Domps, Yaëlle Gorin, Xavier Guillet and Délia Morchoisne

1180. Can pro-growth policies lift all boats? An analysis based on household disposable income (December 2014) by Orsetta Causa, Alain de Serres and Nicolas Ruiz

1179. Empirical evidence on the effects of environmental policy stringency on productivity growth (December 2014) by Silvia Albrizio, Tomasz Koźluk and Vera Zipperer

1178. The Indicators of the Economic Burdens of Environmental Policy Design - Results from the OECD Questionnaire

(December 2014) by Tomasz Koźluk

1177. Measuring Environmental Policy Stringency in OECD Countries-A Composite Index Approach (December 2014) by Enrico Botta and Tomasz Koźluk

1176. Do Environmental Policies Matter for Productivity Growth? Insights from new Cross-Country Measures of Environmental Policies

(December 2014) by Silvia Albrizio, Enrico Botta, Tomasz Koźluk and Vera Zipperer

1175. Making economic growth more socially inclusive

(December 2014) by Andrés Fuentes Hutfilter and Andreas Kappeler

1174. New tax and expenditure elasticity estimates for EU budget surveillance

(December 2014) by Robert W.R. Price, Thai-Thanh Dang and Yvan Guillemette

1173. Moving towards a more dynamic business sector in Spain

(November 2014) by Alberto Gonzalez Pandiella

1172. Better harnessing talent and knowledge to boost sustainable medium-growth in Spain

(November 2014) by David Haugh and Ben Westmore

1171. The internet economy - regulatory challenges and practices

(November 2014) by Isabell Koske, Rosamaria Bitetti, Isabelle Wanner and Ewan Sutherland

1170. A revival of the private rental sector of the housing market? Lessons from Germany, Finland, the Czech Republic and the Netherlands

(October 2014) by Rik de Boer and Rosamaria Bitetti

1169. Secular stagnation: evidence and implications for economic policy

(October 2014) by Łukasz Rawdanowicz, Romain Bouis, Kei-Ichiro Inaba and

Ane Kathrine Christensen

1168. Investment gaps after the crisis

(October 2014) by Christine Lewis, Nigel Pain, Jan Strasky and Fusako Menkyna 
1167. Factors behind the decline in real long-term government bond yield

(October 2014) by Romain Bouis, Kei-Ichiro Inaba, Łukasz Rawdanowicz and

Ane Kathrine Christensen

1166. The effect of the global financial crisis on the OECD potential output

(October 2014) by Patrice Ollivaud and David Turner

1165. Determinants of households' investment in energy efficiency and renewables - evidence from the OECD Survey on household environmental behaviour and attitudes

(October 2014) by Nadia Ameli and Nicola Brandt

1164. Addressing high household debt in Korea

(September 2014) by Randall S. Jones and Myungkyoo Kim

1163. Reducing the high rate of poverty among the elderly in Korea

(September 2014) by Randall S. Jones and Satoshi Urasawa

1162. Promoting the financing of SMEs and start-ups in Korea

(September 2014) by Randall S. Jones and Myungkyoo Kim

1161. Fostering inclusive growth by promoting structural change in the business sector

(September 2014) by Rauf Gönenç, Oliver Röhn, Vincent Koen and Fethi Öğünç

1160. Reducing macroeconomic imbalances in Turkey

(September 2014) by Oliver Röhn, Rauf Gönenç, Vincent Koen and Evren Erdoğan Coşar

1159. Reinvigorating the EU Single Market

(September 2014) by Jean-Marc Fournier.

1158. An exploration of the determinants of the subjective well-being of Americans during the great recession

(August 2014) by Aida Caldera Sánchez and Caroline Tassot.

1157. Boosting the development of efficient SMEs in the Netherlands

(September) by Rafał Kierzenkowski and Jochebed Kastaneer

1156. Making the banking sector more resilient and reducing household debt in the Netherlands

(September 2014) by Rafał Kierzenkowski, Olena Havrylchyk and Pierre Beynet

1155. US long term interest rates and capital flows to emerging economies

(July 2014) by Eduardo Olaberria

1154. Productivity measurement with natural capital and bad outputs

(July 2014) by Nicola Brandt, Paul Schreyer and Vera Zipperer

1153. Reducing income inequality and poverty and promoting social mobility in Korea (July 2014) by Randall S. Jones and Satoshi Urasawa

1152. Fostering a creative economy to drive Korean growth (July 2014) by Randall S. Jones and Myungkyoo Kim 